\title{
ABBA the new face of ACID
}

Helmut Neumann ${ }^{1}$ and Raf Bisschops ${ }^{2}$

${ }^{1}$ Department of Medicine I, University Medical Mainz, Germany

2Department of Gastroenterology and Hepatology, University Hospitals Leuven, TARGID, KU Leuven, Leuven, Belgium

Corresponding author:

Raf Bisschops, MD, PhD

Professor of Medicine

Department of Gastroenterology and Hepatology, University Hospitals Leuven Herestraat 49

3000 Leuven, Belgium

raf.bisschops@uzleuven.be 
If you change your mind, I'm the first in line. Honey I'm still free. Take a chance on me.

Take A Chance On Me, Abba, 1977

Within recent years we have seen an impressive development of advanced endoscopic imaging modalities helping us to better diagnose luminal gastrointestinal diseases and guiding subsequent (endoscopic) therapy [1]. With the evolvement of those new techniques, a continuous shift from the pathologist to the endoscopist seems to occur in the responsibility to make a better pathological diagnosis.

Nevertheless, although the approach of optical biopsies has nowadays been well established for colorectal polyps, non-targeting mapping biopsies (Seattle protocol) are still the standard method for surveillance of patients with Barrett's in all available guidelines [2]. In contrast to, generally well recognizable colon polyps, the majority of neoplasia in Barrett's is flat and often not visible with standard white-light endoscopy alone. Only about $13 \%$ of early neoplasia in Barrett's appears as macroscopically visible nodules. Since lesions in the presence of dysplasia are already often missed, accordingly, detection of subtle lesions in a surveillance setting remains still the greatest challenge [3].

Although the need of surveillance gastroscopy in Barrett's is obvious, mapping biopsies according to the Seattle protocol have major limitations. Like every nonsystematic and non-targeted approach in endoscopy, mapping biopsies are time consuming, expensive and less accepted by both patients and endoscopists. The later only follow the recommendation of the Seattle-protocol in up to $50 \%$ of cases [4]. Therefore, different techniques for surveillance of Barrett's patients are highly advocated and the path that was followed in studies for surveillance of longstanding 
colitis to abandon random biopsies should also be followed for Barrett's surveillance [5].

Chromoendoscopy with acetic acid has shown its effectiveness for neoplasia detection in high-risk Barrett's populations [6]. Acetic acid results in a reversible acetowhitening of the Barrett's mucosa by causing acetylation of cytoplasmic proteins. Neoplastic areas have reduced cytoplasmic proteins causing an early loss of acetowhitening. Accordingly, after acetic acid application to the Barrett's mucosa, neoplastic areas appear "red". Advantages of acetic acid include its universe availability (e.g. vinegar from the grocery store) and the short learning curve in interpreting the images (just evaluate for the "red-in-white-sign") [6]. However, despite its advantages, no randomized controlled trials comparing acetic acid chromoendoscopy to standard mapping biopsies are available yet.

In this issue of Endoscopy, the group of Prof. Bhandari presented the results of the multicenter randomized ABBA-trial, comparing neoplasia detection rates of nontargeted biopsies (Seattle protocol) versus acetic-acid targeted biopsies [7]. With this trial, the authors introduced a new tune in studying advanced imaging in Barrett's esophagus, since it was performed in a standard surveillance setting and to assess accurately the power and acceptability of a back-to-back study with two separate endoscopies.

The study included six centers, from university hospitals to smaller district hospitals and included 200 patients under Barrett's surveillance without a history of neoplasia. In a crossover trial, patients were randomized to either the Seattle protocol or aceticacid targeted biopsies first. 6-8 weeks after the first gastroscopy, $87 \%$ of patients agreed to participate in the second gastroscopy thereby serving as their own control. 
To avoid bias, the pathologist did not release the histology report (unless cancer was detected) until both endoscopies had been performed.

Longcroft-Wheaton et al. showed a zero miss-rate for high-risk neoplasia. Notable, low-grade intraepithelial neoplasia was missed by both approaches. Interestingly, all of those cases have not been found on follow-up gastroscopy and biopsy thereby potentially highlighting the low interobserver agreement of pathologists for diagnosing low-grade Barrett's dysplasia. Up to 12 biopsies per patients were required with the Seattle protocol to diagnose neoplasia, while targeted-biopsies after dye-spraying only required 1.2 biopsies per patient. Based on this data, the authors also provided a cost-effectiveness analysis for the U.K., showing a 9.5 difference between nontargeted and targeted biopsies.

Therefore, the study adds major knowledge to the current literature and highlights the importance of a well-structured study design and an exploratory trial to accurately power a subsequent randomized trial. As highlighted by the authors, some clinicians included in the study were not comfortable with the approach of not taking any biopsies during the acetic acid evaluation when all Barrett's tissue appeared homogenously "white". However, they felt reassured with the tandem endoscopy design. Indeed, by re-capturing, that a 9.5 decrease of biopsies with the targeted approach has been observed, one can fully appreciate the concern of some endoscopists to overlook important pathology. The ABBA-trial however provides strong data that advanced pathology is not overlooked and therefore now allows for powering of a definitive non-inferiority study. The authors estimated 2828 patients for such a trial and we strongly advocate conducting the study now as soon as possible in order to finally prove the effectiveness of acetic acid chromoendoscopy with targeted biopsies for surveillance of Barrett's patients. 
Coming back to one of the most popular songs of Abba, indeed, we can change our mind and maybe should increasingly use targeted biopsy-approaches instead of nonsystematic non-targeted protocols. We should take the chance on ACID, as the new sound of the future for surveillance in Barrett's. Hopefully, the subsequent trial will provide sufficient evidence to follow the more effective path of targeted biopsies, like in chronic colitis. However, based on the current ABBA-trial one can yet not recommend hasty discontinuation of the Seattle mapping biopsies.

\section{References}

1. Neumann H, Bisschops R. Artificial intelligence and the future of endoscopy. Dig Endosc. 2019;31:389-390.

2. Weusten B, Bisschops R, Coron E, et al. Endoscopic management of Barrett's esophagus: European Society of Gastrointestinal Endoscopy (ESGE) Position Statement. Endoscopy. 2017;49:191-198.

3. Schölvinck DW, van der Meulen K, Bergman JJGHM, et al. Detection of lesions in dysplastic Barrett's esophagus by community and expert endoscopists. Endoscopy. 2017;49:113-120.

4. Shaheen NJ, Falk GW, lyer PG, et al. ACG Clinical Guideline: Diagnosis and Management of Barrett's Esophagus. Am J Gastroenterol. 2016;111:30-50.

5. Bisschops R, Bessissow T, Joseph JA, et al. Chromoendoscopy versus narrow band imaging in UC: a prospective randomised controlled trial. Gut. 2018;67:1087-1094. 
6. Kandiah K, Chedgy FJQ, Subramaniam S, et al. International development and validation of a classification system for the identification of Barrett's neoplasia using acetic acid chromoendoscopy: the Portsmouth acetic acid classification (PREDICT). Gut. 2018;67:2085-2091.

7. To be included by Editorial Office - Publication of Longcroft-Wheaton et al. 